\title{
The radio-X-ray luminosity correlation of radio halos at low radio frequency
}

\section{Application of the turbulent re-acceleration model}

\author{
R. Cassano \\ INAF - Istituto di Radioastronomia, via P. Gobetti 101, 40129 Bologna, Italy \\ e-mail: rcassano@ira.inaf.it \\ Received 8 November 2009 / Accepted 6 April 2010 \\ ABSTRACT

\begin{abstract}
Aims. We show expectations on the radio-X-ray luminosity correlation of radio halos at $120 \mathrm{MHz}$. According to the turbulent reacceleration scenario, we expect that low-frequency observations can detect a new population of radio halos that due to their ultrasteep spectra are missed by present observations at $\sim \mathrm{GHz}$ frequencies. These radio halos are also supposed to be less luminous than presently observed halos hosted in clusters with the same X-ray luminosity.

Methods. With Monte Carlo procedures we show that these ultra-steep spectrum halos at $120 \mathrm{MHz}$ cause a steepening and a broadening of the correlation between the synchrotron power and the cluster X-ray luminosity with respect to that observed at $1.4 \mathrm{GHz}$. Results. We investigate the role of future low-frequency radio surveys and find that the upcoming LOFAR surveys will be able to test these expectations.
\end{abstract}

Key words. radiation mechanisms: non-thermal - galaxies: clusters: general - radio continuum: general - X-rays: general

\section{Introduction}

Radio halos are diffuse synchrotron sources of the intra-cluster medium (ICM) extended on a mega-parsec scale (e.g., Feretti 2005; Ferrari et al. 2008). They provide the most important evidence of non-thermal components (relativistic particles and magnetic fields) mixed with the hot ICM. Galaxy clusters hosting radio halos are always characterized by a non-relaxed dynamical status suggestive of recent or ongoing merger events (e.g., Buote 2001; Schuecker et al 2001; Govoni et al. 2004; Venturi et al. 2008; Giacintucci et al. 2009). Furhermore, the halo-radio power at $1.4 \mathrm{GHz}$ increases with the cluster's X-ray luminosity, mass, and temperature (e.g., Liang et al. 2000; Enßlin \& Röttgering 2002; Bacchi et al. 2003; Clarke 2005; Dolag et al. 2005; Cassano et al. 2006, 2007; Brunetti et al. 2009; Rudnick \& Lemmerman 2009; Giovannini et al. 2009). These correlations and the radio halo-merger connection suggest that gravity provides the reservoir of energy to generate the non-thermal components (e.g., Kempner \& Sarazin 2001). Cluster mergers drive shocks and turbulence in the ICM that may amplify the magnetic fields (e.g., Carilli \& Taylor 2002; Dolag et al. 2002; Brüggen et al. 2005; Subramanian et al. 2006; Ryu et al. 2008) and accelerate high-energy particles (e.g., Fujita et al. 2003; Hoeft \& Brüggen 2007; Brunetti \& Lazarian 2007; Pfrommer et al. 2008; Vazza et al. 2009).

Two main scenarios have been proposed to explain the origin of relativistic particles in radio halos, namely i) the turbulent re-acceleration model, in which relativistic electrons are reenergized in situ due to the interaction with MHD turbulence generated in the ICM during cluster mergers (e.g., Brunetti et al. 2001; Petrosian et al. 2001), and ii) the secondary electron models, in which the relativistic electrons are secondary products of the collisions between cosmic rays and thermal protons in the ICM (e.g., Dennison 1980; Blasi \& Colafrancesco 1999; Pfrommer \& Enßlin 2004).

Observations provide support to the idea that turbulence may play a role in the particle re-acceleration process (e.g., Brunetti et al. 2008; Ferrari et al. 2008; Cassano 2009; Giovannini et al. 2009), in which case the population of radio halos is predicted to be a mixture of sources with different spectral properties, with halos with steeper spectra in the majority (Cassano et al. 2006, hereafter C06; Cassano et al. 2009, hereafter C09). That is why upcoming observations with the Low Frequency Array (LOFAR) and the Long Wavelength Array (LWA) will be crucial because very steep spectrum halos should glow up at low radio frequency.

We discuss how such a predicted population is expected to affect the properties of the radio-X-ray luminosity correlation at low radio frequency and investigate the potential of LOFAR surveys. A $\Lambda$ CDM cosmology $\left(H_{0}=70 \mathrm{~km} \mathrm{~s}^{-1} \mathrm{Mpc}^{-1}, \Omega_{\mathrm{m}}=\right.$ $\left.0.3, \Omega_{\Lambda}=0.7\right)$ is adopted.

\section{The population of ultra-steep spectrum radio halos}

The formation and evolution of radio halos according to the turbulent re-acceleration scenario have been investigated by means of Monte Carlo based procedures (Cassano \& Brunetti 2005; C06; C09). These procedures allow us to account for the main components of the model, i.e., the rate of cluster-cluster mergers in the Universe, their mass ratio, and the fraction of energy dissipated during mergers that is channelled into MHD turbulence and acceleration of relativistic particles. We simulate the formation history of $\sim 1000$ galaxy clusters with present day-masses in the range $\sim[0.2-6] \times 10^{15} M_{\odot}$. 
Turbulence acceleration is a rather inefficient process in the ICM and electrons can be accelerated up to energy of several $\mathrm{GeV}$, because at higher energy the radiation losses dominate (e.g., Brunetti \& Lazarian 2007). This implies a gradual spectral steepening at high frequencies in the synchrotron spectrum of radio halos. This steepening makes it difficult to detect radio halos at frequencies higher than the frequency $v_{\mathrm{s}}$ at which the steepening becomes severe.

Following C06 and C09 we use homogeneous models that assume $i$ ) an average value of the magnetic field strength in the radio-halo volume that scales with the cluster mass as $B=$ $B_{\langle M\rangle}\left(M_{\mathrm{v}} /\langle M\rangle\right)^{b 1}$, ii) that a fraction, $\eta_{\mathrm{t}}$, of the $P \mathrm{~d} V$ work, done by subclusters crossing the main clusters during mergers, goes into magneto-acoustic turbulence. The frequency $v_{\mathrm{s}}$, defined as the frequency at which the spectral slope of the halos becomes $\alpha \geq 1.9\left(P(v) \propto v^{-\alpha}\right)$, depends on the acceleration efficiency in the ICM, $\chi$, on the magnetic field in the ICM, $B$, and on the energy density of the cosmic microwave background radiation $(\mathrm{CMB})$ as: $v_{\mathrm{s}} \propto\left(B \chi^{2}\right) /\left(B^{2}+B_{\mathrm{cmb}}^{2}\right)^{2}$. The frequency $v_{\mathrm{s}}$ is a more practical re-definition of the synchrotron-break frequency, $v_{\mathrm{b}}$, and is $v_{\mathrm{s}} \sim 7 v_{\mathrm{b}}$ in homogeneous models (C09). According to $\mathrm{C} 09$, for a single major merger between a cluster of mass $M_{\mathrm{v}}$ and a subcluster with mass $\Delta M, v_{\mathrm{s}}$ is

$$
v_{\mathrm{s}} \propto \frac{B\left(k_{\mathrm{B}} T\right)^{-1}}{\left(B^{2}+B_{\mathrm{cmb}}^{2}\right)^{2}}\left(\frac{M_{\mathrm{v}}+\Delta M}{R_{\mathrm{v}}}\right)^{3},
$$

where $B_{\mathrm{cmb}}=3.2(1+z)^{2} \mu G$ is the equivalent magnetic field strength of the CMB, and $R_{\mathrm{v}}$ is the cluster virial radius. It is expected that mergers may generate halos with higher $v_{\mathrm{S}}$ in more massive clusters, and that halos in clusters with the same mass $M_{\mathrm{v}}$ (and magnetic field) and redshift could have different $v_{\mathrm{S}}$ depending on the properties of the merger event responsible for their generation. Halos with $v_{\mathrm{s}} \geq 1.4 \mathrm{GHz}$ must be generated in connection with the most energetic merger-events in the Universe because only these mergers may allow for the efficient acceleration that is necessary to have relativistic electrons emitting at these frequencies. Present surveys carried out at $v_{0} \sim$ $1 \mathrm{GHz}$ detect radio halos only in the most massive and merging clusters (e.g., Buote 2001; Venturi et al. 2008). On the other hand, radio halos with lower values of $v_{\mathrm{s}}$ must be more common, because they can be generated in connection with less energetic phenomena, e.g., major mergers between less massive systems or minor mergers in massive systems, which are more frequent in the Universe. This has been addressed quantitatively by means of Monte Carlo calculations, which allow us to derive the fraction of clusters with radio halos with different $v_{\mathrm{s}}$ as a function of the cluster mass and redshift. The expected population of radio halos is indeed constituted by a mixture of halos with different spectra, with steep spectrum halos being more common in the Universe; 200 radio halos with $120<v_{\mathrm{s}}<600 \mathrm{MHz}$ are expected in future LOFAR surveys at $120 \mathrm{MHz}$ (C09).

According to this model the monochromatic luminosity of radio halos at a given frequency $v_{0}<v_{\mathrm{s}}$ increases with increasing $v_{\mathrm{s}}$. For a fixed cluster mass (or X-ray luminosity), the relation between the radio luminosity at $v_{0}$ of halos with $v_{\mathrm{S}}=v_{\mathrm{s}, 1}$ and $v_{\mathrm{s}, 2}, P_{v_{\mathrm{s}, 1}}\left(v_{0}, L_{\mathrm{X}}\right)$ and $P_{v_{\mathrm{s}, 2}}\left(v_{0}, L_{\mathrm{X}}\right)$ respectively, is (C09)

$P_{v_{\mathrm{s}, 1}}\left(v_{0}, L_{\mathrm{X}}\right)=P_{v_{\mathrm{s}, 2}}\left(v_{0}, L_{\mathrm{X}}\right)\left(\frac{v_{\mathrm{s}, 1}}{v_{\mathrm{s}, 2}}\right)^{\alpha}$,

where $\alpha \approx 1.3$ (e.g., Ferrari et al. 2008) is the radio spectral index. A correlation between the radio power $P_{\mathrm{R}}$ of halos and the

\footnotetext{
${ }^{1} B_{\langle M\rangle}$ is the value of the magnetic field averaged in a region of radius $=500 h_{50}^{-1} \mathrm{kpc}$ in a cluster with viral mass $\langle M\rangle=1.6 \times 10^{15} M_{\odot}$.
}

mass (and X-ray luminosity) of the hosting clusters is expected (C06). In the simplest case that halos are generated by a single major merger this is

$P_{\mathrm{R}} \propto \frac{M_{\mathrm{v}}^{2-\Gamma} B^{2}}{\left(B^{2}+B_{\mathrm{cmb}}^{2}\right)^{2}}$,

where the parameter $\Gamma$ is defined by $T \propto M^{\Gamma}(\Gamma \simeq 2 / 3$ in the virial scaling). Equation (3) implies that more massive clusters host more luminous radio halos. By considering halos with $v_{\mathrm{s}} \geq 1.4 \mathrm{GHz}, \mathrm{C} 06$ showed that the slope of this scaling is consistent with that of the observed $P(1.4)-M_{\mathrm{v}}$ correlation, provided that the model parameters $\left(B_{\langle M\rangle}, b, \eta_{\mathrm{t}}\right)$ lie within a fairly constrained range of values (see Fig. 7 in C06). We refer the reader to Sects. 3.3 and 4.1 of $\mathrm{C} 06$ for a more detailed discussion on model parameters and their constraints.

Following C09, we adopt a reference set of parameters ${ }^{2}$ : $B_{\langle M\rangle}=1.9 \mu \mathrm{G}, b=1.5, \eta_{\mathrm{t}}=0.2$ that falls in that range and sets $\alpha_{\mathrm{M}}=3.3$, with $P(1.4) \propto M_{\mathrm{v}}^{\alpha_{\mathrm{M}}}$. This implies $P(1.4) \propto L_{\mathrm{X}}^{2.25}$ assuming the $L_{\mathrm{X}}-M_{\mathrm{V}}$ correlation for galaxy clusters as derived in C06. Because the bulk of radio halos in our calculations is found to be associated with clusters of mass $\sim[1-2] \times 10^{15} M_{\odot}$ the adopted values of $B$ and $b$ imply typical average magnetic fields $\sim 1-3 \mu \mathrm{G}$. These values of $B$ are similar to those derived from rotation measurements (e.g., Govoni \& Feretti 2004; Bonafede et al. 2010) and equipartition assumption (Enßlin et al. 1998; Govoni et al. 2001).

The observed $P(1.4)-L_{\mathrm{X}}$ correlation shows an intrinsic scatter across the radio luminosity $\delta P / P \simeq \pm 2$ (e.g., Brunetti et al. 2009). In principle, in our model a scatter in the $P(1.4)-L_{X}$ correlation is expected due to the different monochromatic radio luminosity of halos with different $v_{\mathrm{s}}$ (Eq. (2)). Our calculations show that the fraction of clusters hosting radio halos with $v_{\mathrm{s}} \geq 3500 \mathrm{MHz}$ is about a few percent, thus we would expect $\delta P / P= \pm 1 / 2 \times(3500 / 1400)^{1.3} \sim 1.7$ for halos with $v_{\mathrm{s}} \geq 1.4 \mathrm{GHz}$, which agrees with the observed scatter. However, we stress that there are other possible sources of scatter which are difficult to take into account in homogeneous models. These are due to e.g., differences in the cosmic rays and magnetic field content in clusters with the same mass.

Once we anchor the luminosity of halos with $v_{\mathrm{s}}>1.4 \mathrm{GHz}$, $P_{1.4}\left(1.4, L_{\mathrm{X}}\right)$, to the observed $P(1.4)-L_{\mathrm{X}}$ correlation, Monte Carlo calculations carried out by considering an observing frequency $v_{0}$ and Eq. (2) allow us to derive the expected radio halo luminosity functions (RHLF; see e.g., C06 \& C09 for details). As an example, Fig. 1 shows the total RHLF obtained from Monte Carlo calculations at $v_{0}=120 \mathrm{MHz}$ (black line) and $z=0-0.1$, together with the differential contributions to the RHLF from halos with different $v_{\mathrm{s}}$ (see figure caption). As expected, radio halos with smaller $v_{\mathrm{S}}$ mainly contribute to the low-power end of the total RHLF, and the peaks of the RHLF of different populations move towards low radio powers with decreasing $v_{\mathrm{s}}$. This implies that depending on their sensitivity, surveys at low radio frequency will unveil new populations of halos.

\section{Monte Carlo distributions of radio halos in the $P(120)-L_{x}$ plane}

The aim of this section is to investigate how the new population of ultra-steep spectrum halos, predicted in deep low frequency

\footnotetext{
2 We note that for this particular configuration of parameters even for the re-acceleration phase the energy of magnetic field is always dominant with respect to that of relativistic electrons.
} 


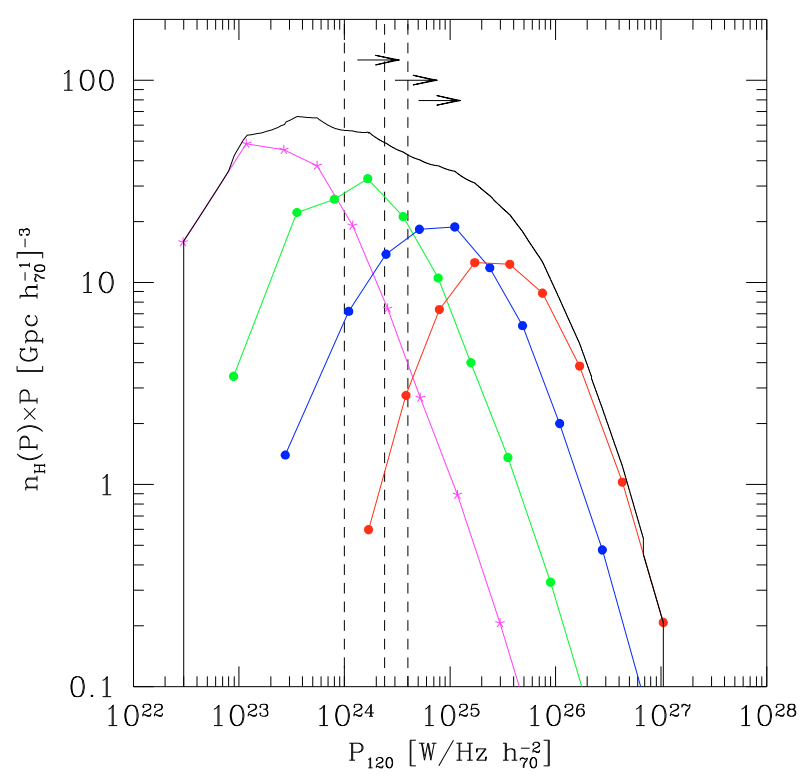

Fig. 1. Radio halo luminosity function at $120 \mathrm{MHz}$ (black lines) and in the redshift interval $z=0-0.1$. The differential contributions from halos with $120<v_{\mathrm{s}}<240 \mathrm{MHz}$ (magenta line, star symbols), $240<$ $v_{\mathrm{s}}<600 \mathrm{MHz}$ (green line), $600<v_{\mathrm{s}}<1400 \mathrm{MHz}$ (blue line) and $v_{\mathrm{s}}>$ $1400 \mathrm{MHz}$ (red line) are also shown. The dashed lines correspond to the minimum detectable halo power for $\xi \cdot F=0.25,0.6$ and $1 \mathrm{mJy} / \mathrm{beam}$ (from left to right, see text).

radio surveys, may affect the radio-X-ray luminosity correlation of halos at low radio frequency. LOFAR will carry out surveys between 15 and $210 \mathrm{MHz}$ in the Northern hemisphere with unprecedented sensitivity and spatial resolution. Because LOFAR is expected to carry out the deepest large area radio surveys at $v_{0}=120 \mathrm{MHz}$ (e.g., Röttgering et al. 2006), we focus here on the $P(120)-L_{\mathrm{X}}$ correlation.

The most crucial point in this respect is the estimate of the minimum diffuse flux of a radio halo detectable by these surveys as a function of redshift. It is well known that the brightness profiles of radio halos smoothly decreases with the distance from the cluster center (e.g., Govoni et al. 2001; Murgia et al. 2009), implying that the outermost region of the halos will be difficult to detect in radio surveys. Brunetti et al. (2007) found that the typical profiles of radio halos are such that about $58 \%$ of their flux is contained within the half radius $\left(R_{\mathrm{H}}\right)$. Following C09 we assume a beam of $25 \times 25$ arcsec to have a good sensitivity to diffuse emission and estimate the minimum flux of a Mpc-sized halo detectable in a LOFAR survey, $f_{\mathrm{H}}$, by requiring that the mean brightness within the half radius of a halo, $B_{<0.5 R_{\mathrm{H}}}$, is $\xi$ times the rms $(F)$ of the survey, i.e.,

$$
\begin{aligned}
B_{<0.5 R_{\mathrm{H}}} & \simeq \frac{0.58 f_{\mathrm{H}}}{\pi / 4 \theta_{\mathrm{H}}^{2}}=\xi F \Rightarrow \\
f_{\mathrm{H}}(z) & \simeq 2 \times 10^{-3}\left[\frac{\xi F}{\mathrm{mJy} / \mathrm{b}}\right]\left[\frac{\theta_{\mathrm{H}}^{2}(z)}{\operatorname{arcsec}^{2}}\right] \mathrm{mJy},
\end{aligned}
$$

where $\theta_{\mathrm{H}}(z)$ is the angular size of radio halos in arcseconds at a given redshift. For $\xi \simeq 1$ this approach would guarantee the detection at several $\sigma$ of the central brightest region of halos, thus leading to the identification of candidate radio halos in the survey.

This simple approach has been tested in Cassano et al. (2008) by injecting "fake" radio halos in the $(u, v)$ plane of NVSS and GMRT observations. It has been shown that radio halos become visible in the images as soon as their flux approaches that obtained by Eq. (4) with $\xi=1$ and $\xi=2$ for the NVSS and GMRT observations, respectively (see also Brunetti et al. 2007; Venturi et al. 2008).

The LOFAR Large Area Survey is expected to reach an $\mathrm{rms} \sim 0.1 \mathrm{mJy} /$ beam at $120 \mathrm{MHz}$ with a beam $\sim 5 \times 5$ arcsec. This resolution and sensitivity is obtained by assuming a "uniform weighting", thus we may assume that the same sensitivity can be reached with a larger beam, obtained for example by e.g., "natural weighting". However, the survey sensitivity may be limited by the rms confusion level. This is given by (e.g., Condon 1987; Kronberg et al. 2007)

$\sigma_{\text {conf }} \simeq 0.13 \times\left[\frac{\theta_{1} \times \theta_{2}}{25 \times 25 \operatorname{arcsec}^{2}}\right] \times\left(\frac{v}{120 \mathrm{MHz}}\right)^{-0.7} \mathrm{mJy}$

where $\theta_{1,2}$ is the beam size in arcsec, and $v$ is the frequency in $\mathrm{MHz}$.

Of course all the issues discussed above will be clarified during the commissioning phase of LOFAR. Thus we decided to present calculations for several cases, specifically $\xi \cdot F=0.25$, 0.6 and $1 \mathrm{mJy} / \mathrm{beam}$ to cover a range of possible LOFAR sensitivities $^{3}$. Vertical dashed lines in Fig. 1 show the minimum power of a halo at $z \sim 0.05$ detectable by LOFAR surveys assuming $\xi \cdot F=0.25,0.6$ and $1 \mathrm{mJy} /$ beam. The important point is that with increasing survey sensitivity new populations of radio halos are expected to be detected, with the detectable number of ultra steep spectrum halos increasing in deeper surveys.

The LOFAR observations will allow us to study the distribution of radio halos in the radio-X-ray luminosity diagram at low radio frequencies, so far an unexplored issue. The vast majority of ultra-steep spectrum halos visible at low frequencies are expected to be associated with galaxy clusters of intermediate $\mathrm{X}$-ray luminosity, $L_{\mathrm{X}} \sim 3-6 \times 10^{44} \mathrm{erg} / \mathrm{s}$, and should be less luminous than radio halos that are presently observed at $\mathrm{GHz}$ frequencies. This should affect the radio-X-ray luminosity correlation of halos at low frequencies, which is expected to be steeper and have a larger scatter than that at $1.4 \mathrm{GHz}$.

To address this issue quantitatively we assumed $v_{0}=$ $120 \mathrm{MHz}$, and following C06 and C09 used Monte Carlo procedures based on the extended Press \& Schechter (1974; Lacey \& Cole 1993) formalism to obtain $i$ ) the population of galaxy clusters, with their mass (and X-ray luminosity), in the redshift interval $z=0-0.5$, and $i i)$ the population of radio halos, with their $v_{\mathrm{S}}$, associated with these clusters. We used homogeneous models and the set of model parameters given in the previous section. From these simulations we extracted the population of radio halos that can be detected by observations at $v_{0}=120 \mathrm{MHz}$ according to their radio luminosity and $f_{\min }(z)$.

In particular, the luminosity at $120 \mathrm{MHz}$ of radio halos with $v_{\mathrm{s}} \geq 1.4 \mathrm{GHz}$ in clusters with $\mathrm{X}$-ray luminosity $L_{\mathrm{X}}$ is obtained from the $P(1.4)-L_{\mathrm{X}}$ correlation, assuming a spectral in$\operatorname{dex} \alpha=1.3$ and allowing for a random scatter $\delta P / P= \pm 2$ (see discussion in Sect. 2). The luminosity at $120 \mathrm{MHz}$ of radio halos with a given $v_{\mathrm{s}}$ is obtained according to Eq. (2). In particular, we calculated halo statistics by assuming the following frequency ranges: $v_{\mathrm{s}}=120-240 \mathrm{MHz}, 240-600 \mathrm{MHz}, 600-1400 \mathrm{MHz}$. Equation (2) also implies that halos with $v_{1} \leq v_{\mathrm{s}}<v_{2}$ should

\footnotetext{
${ }^{3}$ Note that our choice of $\xi \cdot F$ is thought to mimic different possible configurations, e.g., $\xi \cdot F=0.6 \mathrm{mJy} / \mathrm{beam}$ can be $\xi=3(3 \sigma$ detection of the average halo brightness in half $R_{\mathrm{H}}$ ) and $F=0.2 \mathrm{mJy} / \mathrm{beam}$, or $\xi=1$ and $F=0.6 \mathrm{mJy} / \mathrm{beam}$.
} 

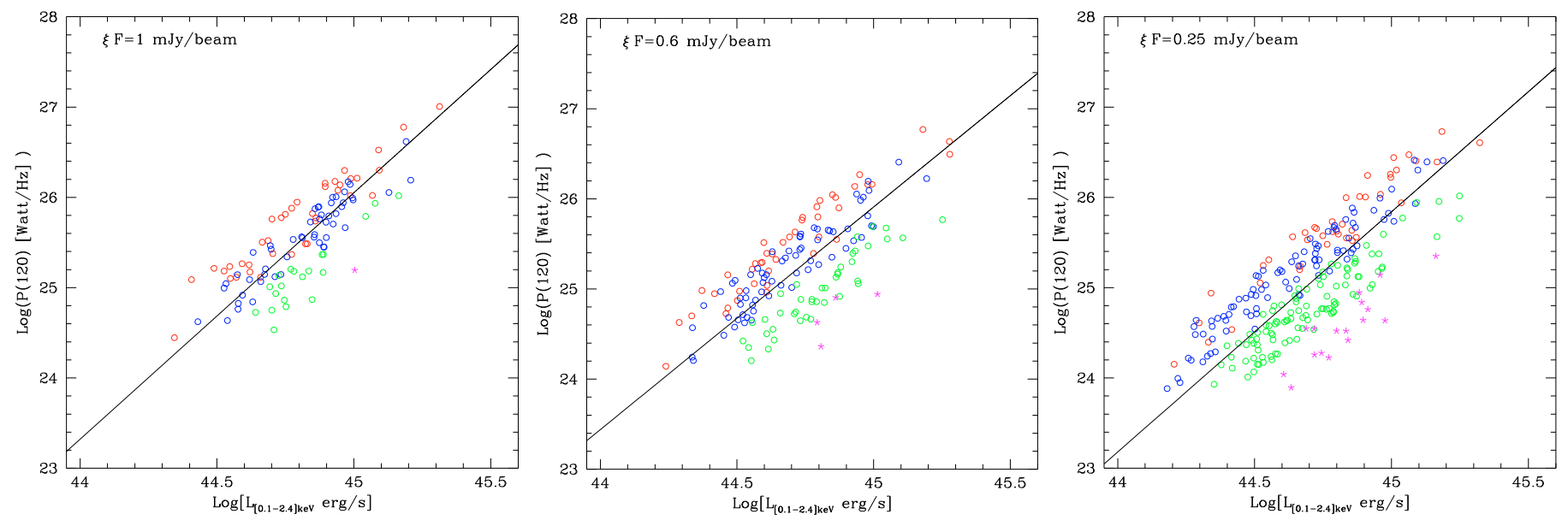

Fig. 2. Expected distribution of radio halos in the $P(120)-L_{\mathrm{X}}$ diagram (colored open dots, the color code is the same as Fig. 1). The correlation $P(1.4)-L_{\mathrm{X}}$ extrapolated at $120 \mathrm{MHz}$ (dashed lines) and the best fit of the $P(120)-L_{\mathrm{X}}$ correlation (solid lines) are also shown. From left to right $\xi F=1,0.6$ and $0.25 \mathrm{mJy} /$ beam.
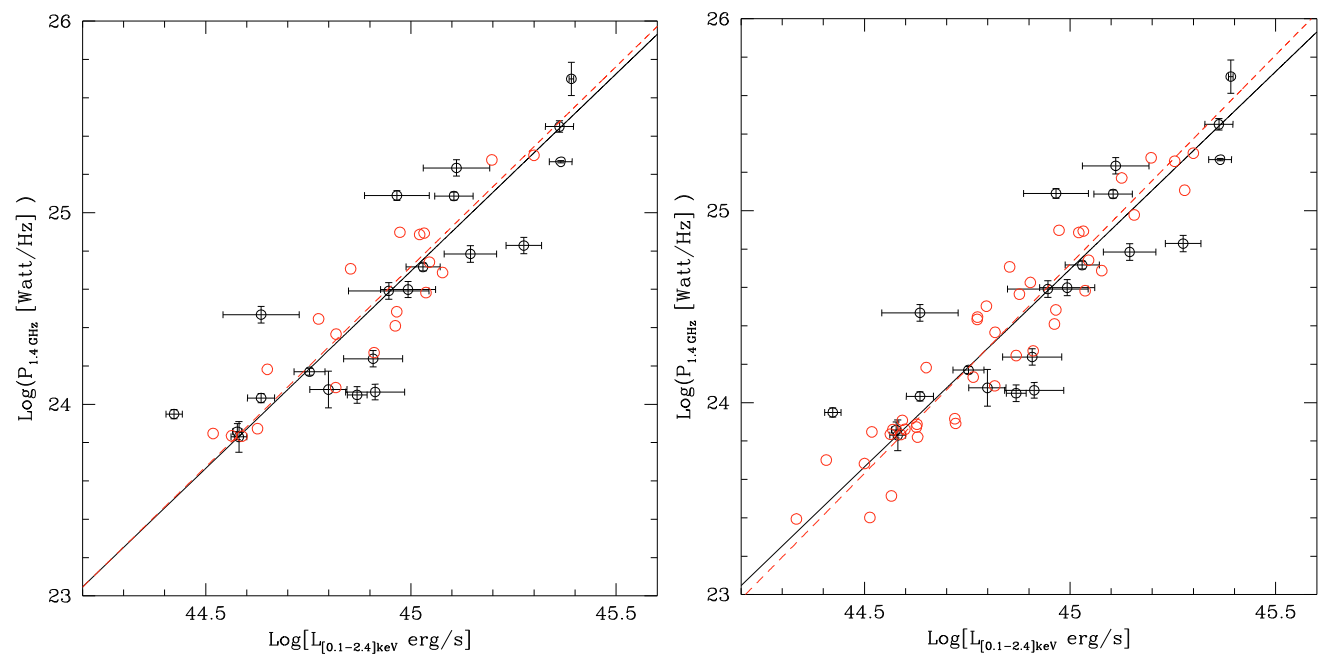

Fig. 3. Radio-X-ray luminosity correlation of giant radio halos at $1.4 \mathrm{GHz}$. Observed halos at $1.4 \mathrm{GHz}$ (black points) and "simulated halos" with $v_{\mathrm{s}}>1.4 \mathrm{GHz}$ (red points) are plotted together with their best-fit relations (solid black line and dashed red line, respectively). Left panel: the observed halos are compared with those expected by the model by considering X-ray and radio sensitivities and sky coverage of GMRT+NVSS samples (see text). Right panel: present observed halos are compared with those with $v_{\mathrm{s}}>1.4 \mathrm{GHz}$, which are expected to be detected by LOFAR surveys assuming $\xi F \simeq 0.25 \mathrm{mJy} / \mathrm{beam}$.

have radio luminosities at $120 \mathrm{MHz}$ which may scatter by a factor $\left(v_{2} / v_{1}\right)^{\alpha}$, which implies $\delta P / P \simeq \pm 1.3-1.7$ for the frequency bins we are considering. Finally, we assumed the LOFAR sky coverage (the Northern hemisphere, $\delta \geq 0$, and high Galactic latitudes, $|b| \geq 20$ ) and $f_{\min }(z)$ from Eq. (4).

The resulting theoretical distribution of radio halos in the $P(120)-L_{\mathrm{X}}$ diagram is shown in Fig. 2, assuming $\xi \cdot F=1,0.6$ and $0.25 \mathrm{mJy} /$ beam (colored open dots; from left to right). Different colored dots indicate halos with different values of $v_{\mathrm{s}}$ (the same color code used in Fig. 1). Halos with different $v_{\mathrm{s}}$ fill different regions, with radio halos with lower $v_{\mathrm{s}}$ typically located in regions of lower radio luminosities. The number of halos with lower $v_{\mathrm{s}}$ increases with increasing survey sensitivity. In highsensitivity surveys these halos dominate the population and their presence affects the overall shape of the correlation. The correlation at $120 \mathrm{MHz}$ is predicted to be more scattered and steeper than that observed at $1.4 \mathrm{GHz}$. A quantitative estimate of the steepening can be obtained by repeating the Monte Carlo procedure described above many times and by fitting the obtained halo distributions in the $P(120)-L_{\mathrm{X}}$ diagram. Figure 4 shows a histogram of the slopes of the correlation obtained after 100 Monte Carlo runs for $\xi F \sim 0.25 \mathrm{mJy} / \mathrm{beam}$. The mean value of the slope is $\alpha_{\text {corr }} \simeq 2.68$, while we find $\alpha_{\text {corr }} \sim 2.45$ and 2.46 for $\xi F \sim 1$ and $0.6 \mathrm{mJy} /$ beam, respectively (with $68 \%$ of values typically within $\Delta \alpha \sim 0.07)$. The values of $\alpha_{\text {corr }}$ are significantly higher than those at $1.4 \mathrm{GHz}$.

For completeness we show in Fig. 3 in the left panel, the theoretical distribution of giant radio halos with $v_{\mathrm{s}}>1400 \mathrm{MHz}$ (red points) in the $P(1400)-L_{X}$ plane together with the observed correlation of halos at $1400 \mathrm{MHz}$ (black points, taken from Brunetti et al. 2009; see Table 1 and references therein). Here we follow C09 (Sect. 3) to compare model expectations and present observations and derive the theoretical distribution of radio halos by considering the combination of the NVSSXBACs (Giovannini et al. 1999) (radio-X-ray) selection criteria and sky coverage (at $z=0.044-0.2$ ), and the X-ray luminosity range and sky coverage of the GMRT radio halo survey (Venturi et al. 2007, 2008) (at $z=0.2-0.32)^{4}$. The observed

${ }_{4}$ The bulk of halos is found in these surveys. 


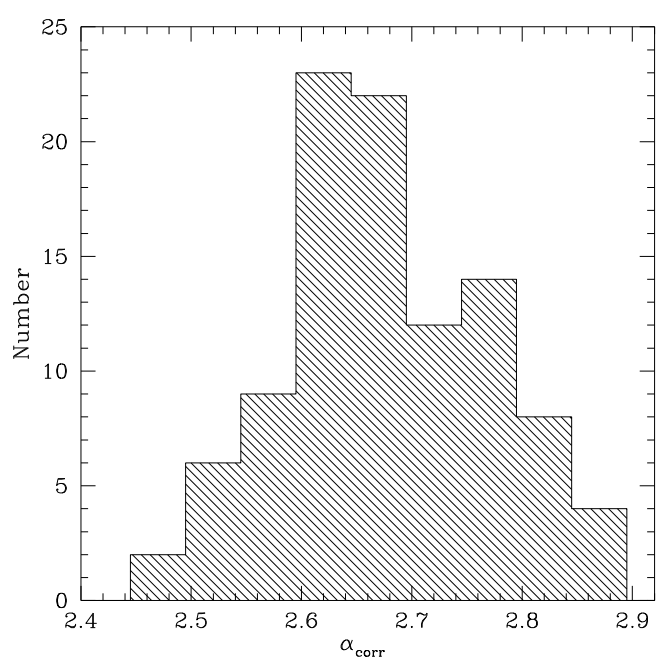

Fig. 4. Spectral slopes of the $P(120)-L_{\mathrm{X}}$ correlation obtained after 100 Monte Carlo extractions of the radio halo distribution in the $P(120)-L_{X}$ diagram, assuming $\xi F=0.25 \mathrm{mJy} / \mathrm{beam}$.

and theoretical distributions agree well, although the data points present a slightly larger scatter than expected, which can be easily interpreted as due to variations of the magnetic field in clusters with the same X-ray luminosity (see Sect. 2). In Fig. 3, right panel, the same observed distribution of radio halos at $1.4 \mathrm{GHz}$ (black points) is compared with that of "simulated" halos with $v_{\mathrm{s}}>1400 \mathrm{MHz}$ (red points) detectable by a LOFAR survey at $120 \mathrm{MHz}$ with $\xi F=0.25 \mathrm{mJy} /$ beam. As expected, radio halos with $v_{\mathrm{s}} \geq 1.4 \mathrm{GHz}$ follow a trend consistent with halos observed at present while their larger number simply reflects the high sensitivity of LOFAR surveys with respect to present surveys.

\subsection{Dependence on model parameters}

The steepening of the correlation is independent of the adopted values of model parameters, at least when considering sets of parameters in the region $\left(B_{\langle M\rangle}, b, \eta_{\mathrm{t}}\right)$ that reproduce both the observed slope of the $P(1.4)-M_{\mathrm{v}}$ correlation $\left(\alpha_{\mathrm{M}}=2.9 \pm 0.4\right)$ and the observed fraction of galaxy clusters with radio halos. Cassano et al. (2006) and C09 already discussed the dependence of the expectations on model parameters. They showed that the expected number of radio halos decreases only by a factor of $\sim 2-2.5$, from super-linear $(b>1)$ to sub-linear $(b<1)$ magnetic scaling (see also Fig. 4 in Cassano et al. 2006).

For a fixed value of $b$ higher values of $B_{\langle M\rangle}$ produce $P(1.4) \propto$ $M_{\mathrm{v}}^{\alpha_{\mathrm{M}}}$ correlations with slightly flatter slopes (see Table 3 in C06). For example, for $b=1.5$ the allowed values of $B$ range from $B_{\langle M\rangle} \simeq 1.9 \mu \mathrm{G}$ to $\simeq 2.8 \mu \mathrm{G}$, and correspondingly it is $\alpha_{\mathrm{M}}=3.3$ and 2.5 , respectively, still consistent with the observed one.

The steepening of the correlation is due to the emergence of new radio halos at low frequency, thus another point is whether the fraction of halos with lower $v_{\mathrm{s}}$ (ultra steep spectrum halos) changes from super-linear to sub-linear cases. To investigate this effect we report in Fig. 5 the percentage of radio halos with $120 \leq v_{\mathrm{s}}<240 \mathrm{MHz}$ (magenta lines) and $600 \leq v_{\mathrm{s}}<1400 \mathrm{MHz}$ (blue lines) as a function of the cluster X-ray luminosity. In Fig. 5 we assume two configurations of parameters: the one used here (solid lines) and a sub-linear one $\left(B_{\langle M\rangle}=0.2 \mu \mathrm{G}\right.$, $b=0.6, \eta_{\mathrm{t}}=0.38$, dashed lines). In both cases, the vast majority of radio halos hosted in clusters with $L_{\mathrm{X}} \lesssim 3 \times 10^{44} \mathrm{erg} / \mathrm{s}$ has $v_{\mathrm{s}} \leq 240 \mathrm{MHz}$, while halos with $v_{\mathrm{s}} \geq 600 \mathrm{MHz}$ become dominant in more luminous clusters. On the other hand, we find that

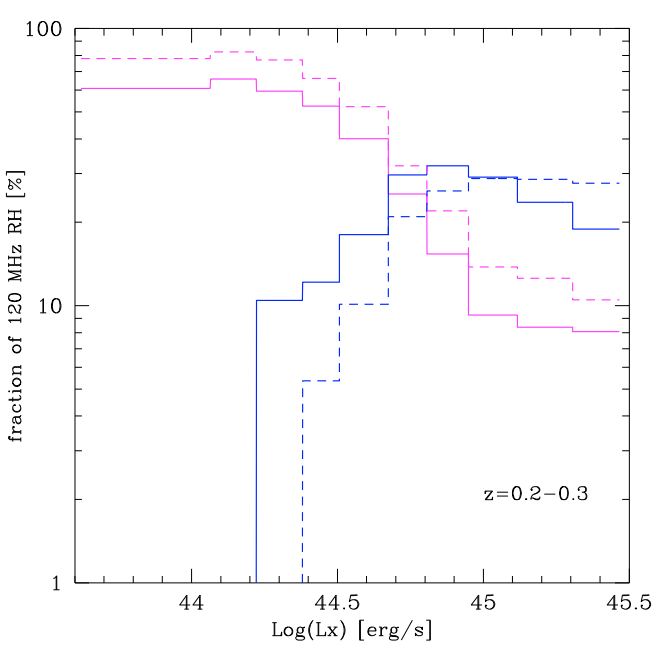

Fig. 5. Fraction of clusters with radio halos with $120 \leq v_{\mathrm{s}}<240 \mathrm{MHz}$ (magenta lines) and $600 \leq v_{\mathrm{s}}<1400 \mathrm{MHz}$ (blue lines) as a function of the cluster X-ray luminosity. The calculations are shown for the redshift range $z=0.2-0.3$ and for $B_{\langle M\rangle}=1.9 \mu \mathrm{G}, b=1.5, \eta_{\mathrm{t}}=0.2$ (solid lines) and $B_{\langle M\rangle}=0.2 \mu \mathrm{G}, b=0.6, \eta_{\mathrm{t}}=0.38$ (dashed lines).

the fraction of halos with $120<v_{\mathrm{s}}<240 \mathrm{MHz}$ is larger in the sub-linear case. This is because it is more difficult to generate radio halos with higher $v_{\mathrm{s}}$ for lower magnetic fields (provided that radiative losses are dominated by the Inverse-Compton losses due to the CMB photons). We may conclude that in sub-linear cases we expect the following main effects: $i$ ) the $P(120)-L_{\mathrm{X}}$ diagram should be less populated than that in super-linear cases (less halos are expected); ii) the $P(1.4)-L_{\mathrm{X}}$ correlation is expected to be flatter than that in super-linear cases (see Table 3 in C06); iii) the $P(120)-L_{\mathrm{X}}$ diagram should be even more dominated by ultra-steep spectrum halos, making the steepening of the correlation at lower frequency even stronger.

\section{The effect of an evolving magnetic field}

We applied a statistical model based on the turbulenceacceleration scenario (discussed and developed in Cassano \& Brunetti 2005; C06; and C09) to derive the expected distribution of radio halos in the $P(120)-L_{\mathrm{X}}$ diagram. The cosmological evolution of the magnetic field in this model is accounted for by scaling the field with the cluster mass, as suggested by cosmological MHD simulations (e.g., Dolag et al. 2002; see also Sect. 2). On the other hand, our calculations do not selfconsistently follow turbulence and the amplification of magnetic fields due to this turbulence. The main reason for that is that in the turbulence acceleration scenario radio halos are generated and disappear due to the acceleration and cooling of the emitting particles, and these processes are much faster ( $~ 0.1-0.2$ Gyr) than the slow decay of the magnetic field in the ICM, few Gyr (e.g., Brunetti et al. 2009). Moreover, although particle-acceleration is demonstrated to be connected with cluster mergers (e.g., Buote 2001; Govoni et al. 2004; Venturi et al. 2008), several mechanisms/sources of magnetic field other than mergers-induced amplification may significantly contribute to the magnetic field in the ICM. Indeed, higher values of magnetic fields are measured in cooling core clusters, which are not merging systems (e.g., Carilli \& Taylor 2002; Govoni 2006). Although it is clear that a self-consistent treatment of turbulence, particle acceleration and magnetic field evolution is mandatory and deserves future theoretical efforts, we show in this section 

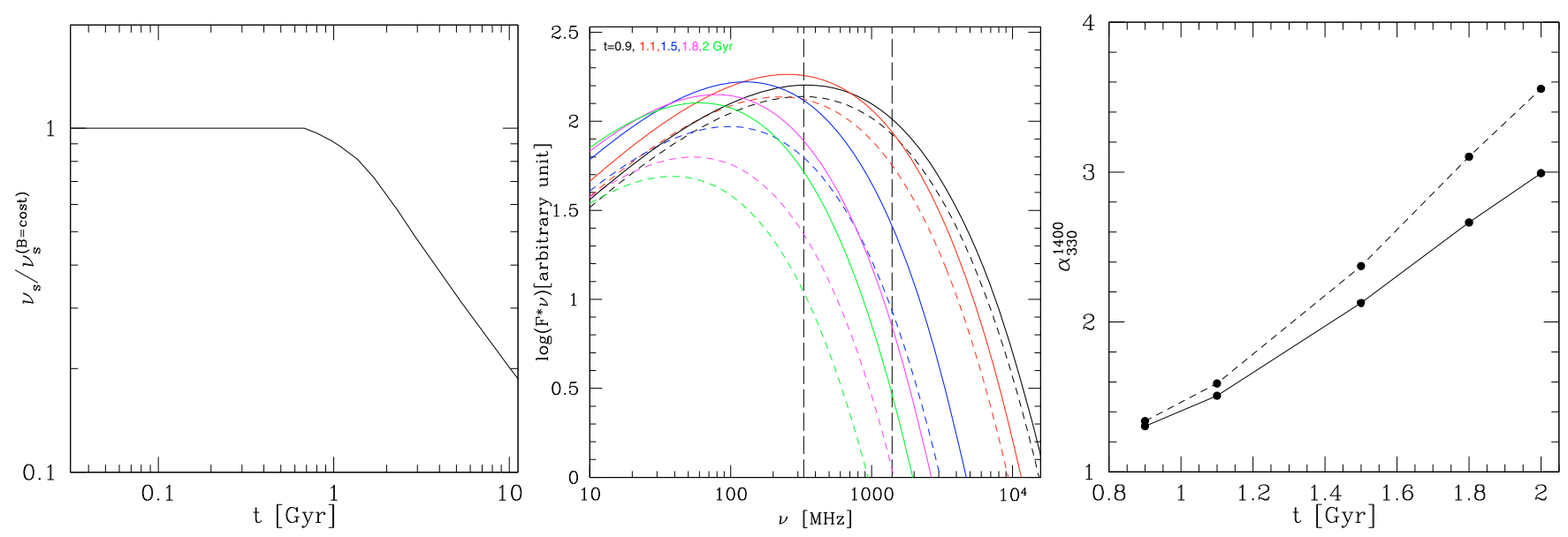

Fig. 6. Left panel: time-evolution of the ratio between $v_{\mathrm{s}}$ computed by assuming an evolving (as in Fig. 2 of SSH06) and a constant magnetic field; central panel: time-evolution of the synchrotron spectra computed by assuming an evolving (dashed lines) and a constant (solid lines) magnetic field. Spectra are reported for $t=0.9,1.1,1.5,1.8$ and $2 \mathrm{Gyr}$ (see color code in the figure panel). Right panel: synchrotron spectral indices between $1.4 \mathrm{GHz}$ and $330 \mathrm{MHz}$ of the synchrotron spectra reported above: for a constant magnetic field (solid line) and for an evolving magnetic field (dashed line).

that the results presented here are not substantially affected by the evolution of the magnetic field when clusters become more relaxed after a merging phase.

In order to investigate the effect of an evolving magnetic field on our results, we consider the simulations of the generation and decay of dynamo-active turbulence developed by Subramanian, Shukurov \& Haugen (2006, hereafter SSH06). They studied the decay phase of an induced turbulent flow and magnetic field that follows a saturation phase after the driving is switched off. They found that after the exponential growth of the magnetic field a saturation phase follows, the turbulent energy and the magnetic energy decay in a way that after an eddy-turnover timescale the turbulent energy density is about half of its saturation value, while the magnetic field strength is still at $\approx 90 \%$ of the saturation value (see Fig. 2 in SSH06). By considering random motion with a typical initial speed $v_{0}=500 \mathrm{~km} \mathrm{~s}^{-1}$ and scale $l_{0} \simeq 300 \mathrm{kpc}$, which are appropriate for cluster-merger driven turbulence, the eddy-turnover timescale is $t_{0} \simeq 0.6 \mathrm{Gyr}$. Because we are interested in the evolution of radio halos on timescales of $\sim \mathrm{Gyr}$ and the radiative lifetime of the emitting particle is of $\sim 0.1-0.3 \mathrm{Gyr}$, we can estimate the variation of the frequency $v_{\mathrm{s}}$ as a function of time by adopting the classical formula $v_{\mathrm{s}} \propto\left(B \chi^{2}\right) /\left(B^{2}+B_{\mathrm{cmb}}^{2}\right)^{2}$ (see also Sect. 2). The ratio between $v_{\mathrm{s}}$ for a time-dependent magnetic field and for a constant magnetic field is reported in Fig. 6 (left panel), where $t_{0}=1.1 \mathrm{Gyr}$ (because here we have considered a saturation phase of $\Delta t=0.5 \mathrm{Gyr}$ before the driving is switched off).

Substantial differences are found only for $t>2$ Gyr when the halo is expected to have already disappeared however $\left(v_{\mathrm{S}} \propto \epsilon_{\mathrm{t}}^{4}\right.$, with $\epsilon_{\mathrm{t}}$ the turbulence energy density). The ratio between the steepening frequencies (Fig. 6, left panel) at $t \sim t_{0}$ gives an estimate of the error we can make by neglecting the time-evolution of the magnetic field, that is $\leq 30 \%$.

In the central panel of Fig. 6 we also report the timeevolution of the synchrotron spectra at different times $(t=0.9$, $1.1,1.5,1.8$ and $2 \mathrm{Gyr}$, see figure caption) by assuming a constant magnetic field (as in the adopted model, solid lines) and an evolving magnetic field (as in SSH06, dashed lines). For $t \leq 1.1$ Gyr the difference in the monochromatic radio luminosities is less then $10 \%$. In addition, the right panel of Fig. 6 shows the evolution of the spectral index between $1.4 \mathrm{GHz}$ and
$330 \mathrm{MHz}$ of these synchrotron spectra. Up to $1.2-1.3 \mathrm{Gyr}$ the difference in the spectral indeces is very small, less then $10 \%$.

\section{Conclusions}

The observed correlations between the halo radio power (at $1.4 \mathrm{GHz}$ ) and the cluster X-ray luminosity, mass, and temperature and the observed connection between radio halos and cluster mergers suggest a link between the gravitational process of cluster formation and the generation of radio halos. Radio halos are likely generated during cluster-cluster mergers where a fraction of the gravitational energy dissipated is channelled into the acceleration of relativistic particles. A crucial expectation of the turbulent re-acceleration scenario, put forward to explain radio halos, is that the synchrotron spectrum of halos is characterized by a cut-off at frequency $v>v_{\mathrm{s}}$ with $v_{\mathrm{s}}$ determined by the efficiency of the acceleration process. This cut-off causes a bias, so that present radio observations at $\sim \mathrm{GHz}$ frequencies are expected to detect only the most efficient radio phenomena in clusters, leaving unexplored a large population of radio halos characterized by a spectral cut-off at lower frequencies (e.g., C06; Brunetti et al. 2008; C09). Future low-frequency radiotelescopes such as LOFAR and LWA are expected to discover the populations of ultra steep spectrum radio halos with $v_{\mathrm{s}}<1 \mathrm{GHz}$, which will enable us to test the idea of turbulent re-acceleration.

One may wonder whether halos with $v_{\mathrm{s}}<1 \mathrm{GHz}$ could be detected by present radiotelescopes at $1.4 \mathrm{GHz}$. To address this point we report in Fig. 7 the flux distribution at $1.4 \mathrm{GHz}$ of halos with $600 \leq v_{\mathrm{s}}<1400 \mathrm{MHz}$ (which in our model have $\alpha \approx 1.7$ between $120 \mathrm{MHz}$ and $1400 \mathrm{MHz}$ ), which are expected to be detected by LOFAR at $120 \mathrm{MHz}$ assuming $\xi F \simeq 0.25 \mathrm{mJy} / \mathrm{beam}$. Calculations are derived by assuming $i$ ) at $z<0.3$ the X-ray flux limit and sky coverage of the extended ROSAT Brightest Cluster Sample (eBCS, Ebeling et al. 1998, 2000) and of the ROSATESO Flux Limited X-ray Galaxy Cluster Survey (REFLEX, Böringher et al. 2004), and ii) at $z=0.3-0.6$ the X-ray flux limit and sky coverage of the Massive Cluster Survey (MACS, Ebeling et al. 2001). Calculations show that potentially very deep pointed observations at $1.4 \mathrm{GHz}$ of all these clusters may lead to the detection of a few of these ultra steep spectrum halos (those with $600 \leq v_{\mathrm{s}}<1400 \mathrm{MHz}$ ); the ultra steep spectrum halo 


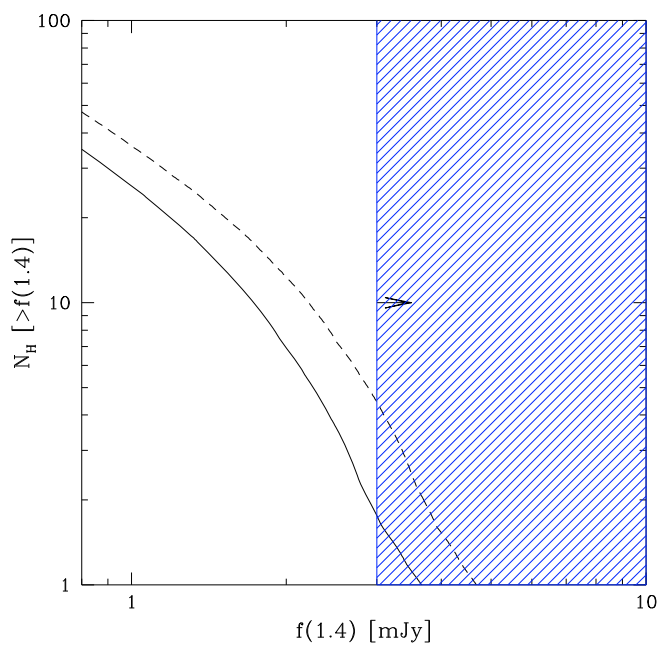

Fig. 7. Integrated number counts at $1.4 \mathrm{GHz}$ of halos with $600 \leq v_{\mathrm{s}}<$ $1400 \mathrm{MHz}$ that are expected to be detected by LOFAR at $120 \mathrm{MHz}$ assuming $\xi F \simeq 0.25 \mathrm{mJy} /$ beam. Calculations are derived by combining $i$ ) at $z<0.3$, the sky coverage and X-ray flux limit of eBCS and REFLEX clusters, and ii) at $z=0.3-0.6$, the sky coverage and X-ray flux limit of MACS clusters. Dashed and solid lines account for the uncertainty due to the finite frequency range of $v_{\mathrm{s}}(600-1400 \mathrm{MHz})$ assumed in the calculations. The dashed blue region shows the typical minimum flux of halos detectable with present radio facilities (e.g., the VLA considering $\mathrm{C}+\mathrm{D}$ configurations) at $1.4 \mathrm{GHz}$ (approximatively $f(1.4)>3-4 \mathrm{mJy}$ ).

detected in the cluster Abell 521 (Brunetti et al. 2008; Dallacasa et al. 2009) belongs to this class of halos, although it is among the flatter spectrum objects in this class, with $v_{\mathrm{s}} \approx 1200 \mathrm{MHz}$.

We discussed the consequence of this new population of radio halos on the slope of the radio-X-ray luminosity correlation at low frequency. According to homogeneous models, ultrasteep spectrum halos are expected to be less luminous than halos with higher $v_{\mathrm{s}}$ associated with clusters of the same mass. Also, radio halos with lower $v_{\mathrm{s}}$ should statistically be generated in clusters with smaller mass (and $L_{X}$ ). The combination of these two expectations implies that the radio-X-ray luminosity correlation should be broader and steeper at lower frequencies.

Based on this model, we performed Monte Carlo simulations of the distribution of radio halos in the $P(120)-L_{\mathrm{X}}$ plane. We found that halos distribute in the $P(120)-L_{\mathrm{X}}$ plane according to a correlation which is steeper $(\Delta \alpha \approx 0.4)$ and broader than that observed at $1.4 \mathrm{GHz}$, with ultra-steep spectrum halos broadening the scatter in the region of low luminosity. We found that the number of ultra-steep spectrum halos increases with increasing survey sensitivity, and this further steepens the correlation. The forthcoming LOFAR surveys should constrain the expected steepening of the correlation and test our expectations.

Although a self-consistent treatment of turbulence acceleration and amplification of the magnetic field in clusters is mandatory and deserves future efforts, we show that the main components in the adopted scenario are two "fast" processes: particle acceleration and particle cooling that follows the decay of turbulence. Because this is a "slow" process, we show that the possible decay of the field with turbulence is not expected to significantly affect the modeling of halo statistics.
Acknowledgements. This work is partially supported by grants PRIN-INAF 2007, PRIN-INAF 2008 and ASI-INAF I/088/06/0. R.C. thanks the anonymous referees for comments and suggestions, and G. Brunetti, M. Brüggen, H. J. A. Röttgering and T. Venturi for useful comments.

\section{References}

Bacchi, M., Feretti, L., Giovannini, G., \& Govoni, F. 2003, A\&A, 400, 465 Blasi, P., \& Colafrancesco, S. 1999, APh, 12, 169 Böhringer, H., Schuecker, P., Guzzo, L., et al. 2004, A\&A, 425, 367 Bonafede, A., Feretti, L., Murgia, M., et al. 2010, A\&A, 513, A30 Brunetti, G., \& Lazarian, A. 2007, MNRAS, 378, 245

Brunetti, G., Setti, G., Feretti, L., \& Giovannini, G. 2001, MNRAS, 320, 365

Brunetti, G., Venturi, T., Dallacasa, D., et al. 2007, ApJ, 670, L5

Brunetti, G., Giacintucci, S., Cassano, R., et al. 2008, Nature, 455, 944 Brunetti, G., Cassano, R., Dolag, K., \& Setti, G. 2009, A\&A, 507, 661 Brüggen, M., Ruszkowski, M., \& Simionescu, A. 2005, ApJ, 631, L21 Buote, D. A. 2001, ApJ, 553, 15

Carilli, C. L., \& Taylor, G. B. 2002, ARA\&A, 40, 319

Cassano, R, 2009, in The Low-Frequency Radio Universe, ed. D. J. Saikia, D. A. Green, Y. Gupta, \& T. Venturi (San Francisco: ASP), ASP Conf. Ser., 407, 223

Cassano, R., \& Brunetti, G. 2005, MNRAS, 357, 1313

Cassano, R., Brunetti, G., \& Setti, G. 2006a, MNRAS, 369, 1577 (C06)

Cassano, R., Brunetti, G., \& Setti, G. 2006b, Astron. Nachr., 327, 557

Cassano R., Brunetti G., Setti G., et al. 2007, MNRAS, 378, 1565

Cassano, R., Brunetti, G., Venturi, T., et al. 2008, A\&A, 480, 687

Cassano, R., Brunetti, G., Rottgering, H. J. A., \& Bruggen M. 2010, A\&A, 509, 68

Clarke, T. E. 2005, ASP Conf. Ser., 345, 227

Condon, J. J. 1987, in Proceedings of the Arecibo Upgrading Workshop, ed. J. H. Taylor, \& M. M. Davis (Arecibo: NAIC), 89

Dallacasa, D., Brunetti, G., Giacintucci, S., et al. 2009, ApJ, 699, 1288

Dolag, K., Bartelmann, M., \& Lesch, H. 2002, A\&A, 387, 383

Dolag, K., Grasso, D., Springel, V., \& Tkachev, I. 2005, JCAP, 1, 9

Dennison, B. 1980, ApJ, 239, L93

Ebeling, H., Edge, A. C., Böhringer, H., et al. 1998, MNRAS, 301, 881

Ebeling, H., Edge, A. C., Allen, S. W., et al. 2000, MNRAS, 318, 333

Ebeling, H., Edge, A. C., \& Henry, J. P. 2001, ApJ, 553, 668

Ensslin, T. A., Biermann, P. L., Klein, U., \& Kohle, S. 1998, A\&A, 332, 395

Enßlin, T. A., \& Röttgering, H. 2002, A\&A, 396, 83

Feretti, L. 2005, in X-Ray and Radio Connections, published electronically by NRAO, ed. L. O. Sjouwerman, \& K. K. Dyer

Ferrari, F., Govoni, F., Schindler, S., et al. 2008, SSRv, 134, 93

Fujita, Y., Takizawa, M., \& Sarazin, C. L. 2003, ApJ, 584, 190

Giacintucci, S., Venturi, T., Brunetti, G., et al. 2009, A\&A, 505, 45

Giovannini, G., Tordi, M., \& Feretti, L. 1999, NewA, 4, 141

Giovannini, G., Bonafede, A., Feretti, L. 2009, A\&A, 507, 1257

Govoni, F. 2006, Astron. Nachr., 327, 539

Govoni, F., \& Feretti, L. 2004, Int. J. Mod. Phys. D, 13, 1549

Govoni, F., Feretti, L., Giovannini, G., et al. 2001, A\&A, 376, 803

Govoni, F., Markevitch, M., \& Vikhlinin, A. 2004, ApJ, 605, 695

Hoeft, M., \& Brüggen, M. 2007, MNRAS, 375, 77

Kempner, J. C., \& Sarazin, C. L. 2001, ApJ, 548, 639

Kronberg, P. P., Kothes, R., Salter, C. J., \& Perillat, P. 2007, ApJ, 659, 267

Lacey, C., \& Cole, S. 1993, MNRAS, 262, 627

Liang, H., Hunstead, R. W., Birkinshaw, M., \& Andreani, P. 2000, ApJ, 544, 686

Murgia, M., Govoni, F., Markevitch, M., et al. 2009, A\&A, 499, 679

Petrosian, V. 2001, ApJ, 557, 560

Pfrommer, C., \& Enßlin, T. A. 2004, A\&A, 413, 17

Pfrommer, C., Enßlin, T. A., \& Springel V. 2008, MNRAS, 385, 1211

Press, W. H., \& Schechter, P. 1974, ApJ, 187, 425

Röttgering, H. J. A., Braun, R., Barthel, P. D., et al. 2006, [arXiv:0610596]

Rudnick, L., \& Lemmerman, J. A. 2009, ApJ, 697, 1341

Ryu, D., Kang, H., Cho, J., \& Das, S. 2008, Science, 320, 909

Schuecker, P., Böhringer, H., Reiprich, T. H., et al. A\&A, 378, 408

Subramanian, K., Shukurov, A., Haugen, N. E. L. 2006, MNRAS, 366, 1437

Vazza, F., Brunetti, G., \& Kritsuk, A. 2009, A\&A, 504, 33

Venturi, T., Giacintucci, S., Brunetti, G., et al. 2007, A\&A, 463, 937

Venturi, T., Giacintucci, S., Dallacasa, et al. 2008, A\&A, 484, 327 